\title{
CURRENT CHALLENGES FOR RESEARCH ACTIVITIES IN THE FIELD OF RAILWAY INFRASTRUCTURE
}

\author{
OtTo PlaseK
}

\begin{abstract}
Brno University of Technology, Faculty of Civil Engineering, Veveri 95, Brno, Czech Republic correspondence: plasek.o@fce.vutbr.cz
\end{abstract}

\begin{abstract}
.
The increasing importance of railway systems, arising from both national and European strategic documents, leads to increasing demands on its infrastructure. Transport development in the European territory is defined in the strategy summarized in the White Paper, which understands the rail sector as a key sector in terms of sustainability of the transport development due to the suppression of congestions, carbon oxide emissions, reliability and safety of transport.

The paper is aimed in the specific technical aspects of rail infrasructure, which emphasize their ability to contribute to an efficient and sustainable transport in Europe and justify motivations for increasing the attractiveness of rail transport. Current challenges for research activities in the field of railway infrastructure that will led to achievement of the objectives which were defined in the White Paper and further specified in the particular requirements of rstakeholders in the railway industry are discussed.
\end{abstract}

KEYWORDS: railway infrastructure, railway research, interoperability of railway infrastructure, noise and vibrations.

\section{INTRODUCTION}

The increasing importance of railway systems, arising from both national and European strategic documents, leads to increasing demands on its infrastructure. Transport development in the European territory is defined in the strategy summarized in the "White Paper Roadmap to a Single European Transport Area - Towards a competitive and resource efficient transport system", which was released by the European Commission in 2011 [1]. This strategy defines the "Roadmap to a Single European Transport Area" with the aim of creating a competitive and efficient transport system. It is evident that an efficient transport systems are crucial for the competiveness of European enterprises in the global economy. This fact is supported by an argument that transport and freight storage costs are $10-15 \%$ of the final products costs. Every European household gives approximately $13.2 \%$ of its budget on transport-related products and services. Annual congestion costs in Europe are approximately $1 \%$ of gross domestic product.

Transport development strategy is based on several obvious assumptions. It is expected a continuous increasing of the oil price which should be at least twice in 2050 compared to 2005, while transport is dependent on oil for at least $96 \%$. Greenhouse gas emission should be reduced $80 \%$ bellow the level of 1990 to limit the global temperature change to $2 \ddot{\mathrm{i} £ j i ̈ £ ; C}$. Alarming is also fact that exceeding of capacity limits for road and air transport requires annually $1 \%$ additional costs in Europe. The increase of freight transport up to $40 \%$ in 2030 and up to $80 \ddot{i} £$; $\%$ in 2050 is assumed. Moreover, the infrastructure is developed unevenly in the eastern and western parts of European Union. Nowadays only about 4,800 km of motorways, no high speed railway lines and conventional railway lines often in poor conditions are in the new Member States [2].

The White Paper for Transport understands the rail sector as a key sector in terms of sustainability of the transport development due to the suppression of traffic congestions, carbon oxide emissions, reliability and safety of transport. The necessity of transformation of rail transport to become more attractive and to increase its market share of passenger and freight transport for medium distance (up to $300 \mathrm{~km}$ ) in 2050 is defined in this strategic plan. In this respect, the White Paper puts a very ambitious goal of moving the road transport to rail or water transport in volume of $50 \%$ [1].

The specific technical aspects of rail transport, which emphasize their ability to contribute to an efficient and sustainable transport in Europe and justify motivations for increasing the attractiveness of rail transport are stated bellow in the paper.

\section{Current role of rail transport}

Rail passenger transport in Europe now plays an important role in long-distance transport (from $300 \mathrm{~km}$ ) through especially high-speed trains [3]. The definition of high-speed services is not easy and involves a wide range factors which determine operator access to the passenger rail transport. Nevertheless, it is usually considered the rail traffic at speeds higher than $230 \mathrm{~km} / \mathrm{h}$. The rail passenger services not quite successfully compete in individual transport for medium 
distances up to $300 \mathrm{~km}$ so far, because the quality of service reflects considerable time losses when access to rail transport and while waiting for the connection or transfer. The exception is train connection in directions where there is no motorway available. That is why rail operators is really interested in the connection Praha - Ostrava. It should be noted that in the mid-range distances will play a crucial role, yet unmentioned aspects of the definition of high-speed traffic, which express the quality of transport:

- Infrastructure (including civil works, tracks, overhead catenary systems, etc.)

- Comfortable railway stations (their location, functional design, equipment, etc.)

- Rolling stock (technically advanced, comfortable, modern design etc.)

- Operation (planning, management, regulations)

- Advanced command and control systems (ETCS)

- Sophisticated strategy of maintenance

- Coherent financing, marketing and management

The suburban transport in big cities or regional centers is another important area of the positive benefits of passenger rail transport. The inclusion of railway transport into integrated transport systems reflects this trend. Rail freight transport currently plays an important role in the field of long distance, especially the transcontinental transport. The effectiveness of the railways in this regard improves the use of combined systems. However, it must be noted that the increase in the medium-range transport (mainly domestic) will always run into tough competition with road transport.

\section{ResEARCH ACTIVItiES IN THE FIELD OF RAILWAY INFRASTRUCTURE}

Research, development and innovation will be focused on construction of high-speed lines, increasing speeds on existing rail lines, increasing reliability and durability of tracks with mixed railway traffic in the context of increasing service load and capacity. The current issue of interoperability of the railway infrastructure is taken into particular consideration.

Rail infrastructure is in the state that corresponds a history of its construction, maintenance, renovation and modernization. Rail infrastructure must be considered as a very complex system, which consists of components that are of different technical, technological advancement and age. Railway track consists both from earthworks such as embankments or cuts and from advanced railway superstructure both ballasted and ballastless, characterized by the controlled vertical stiffness of substructure and with resilient rail fastening. Most of tracks are of different age and technological advancement due to the gradual replacement of the structural elements except completely renewed or modernized tracks.
The main activities of research activities aimed in the development of rail infrastructure, both railway and urban lines are focused on requirements of speed increase, higher service and axle load, safety and security, ride comfort while meeting economic needs, environmental requirements, i.e. reduction of noise, suppression of vibrations spreading, especially in urban areas, also reducing costs of maintenance and repair works, reducing energy consumption and consumption of raw materials. Calls for research projects both national and European correspond to the above specified requirements.

The focus of the research activity in the Czech Republic is the preparation of technological solutions for the design and construction of high-speed railways. An important part of the process is development of decision-making strategies for choosing the most appropriate design solutions for high speed lines (eg. ballasted vs. ballastless track). Research activities are focused on the preparation of new structural and technological solutions to meet the requirements of the railway superstructure and substructure of high-speed lines, especially on the structural design of switches and crossings. The attention must be paid to the particular design of the high speed structures as well as to encourage the readiness of domestic suppliers in railway industry and track work contractors. It is also important to pay attention to the management and maintenance systems of high speed lines, focusing on the question both of logistics and policy-warranty service and preventive maintenance in defined cycles.

Regarding railway substructure, earthworks were constructed usually in the 19 th century. Structural layers ensuring the strength of substructure corresponding to the increased axle load were constructed only for certain lines in the second half of the 20th century. At the turn of the millennium corridor lines have been modernized, where structural layers in the active zone of the embankment have been constructed or completely renewed. Most sections outside the corridor lines does not comply with current requirements due to the age of the earthworks and their original design load. Remedying this situation will require high costs and be associated with a number of construction works, which require service disturbance or interruption. Most of the research is therefore concentrated on finding technologies, increasing strength of substructure especially for weak, compressible or unstable soils. The solutions are searched with help static and dynamic analyses and an optimization of the stiffness of the railway substructure, development of advanced mobile diagnostic methods and tools. Solutions which help to minimize service disturbances and interruptions at the least possible cost of works and consumption of materials are looked for.

Railway superstructure was many times renewed in comparison with rail substructure in the same section. Whenever rail superstructure was usually renewed with up to date technology. This means that except 


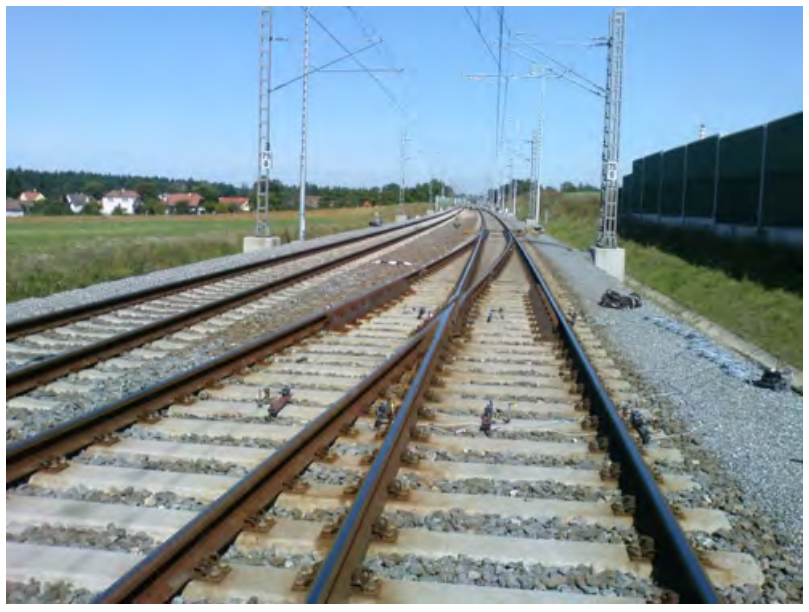

Figure 1. Test section in Plana nad Luznici railway station - under sleeper pads

corridor lines railway superstructure is a mixture of components of different age and technology along the track. It can be state that currently used technology of ballasted tracks are comparable with ones used in high speed lines. Design of switches and crossings $(\mathrm{S} \& \mathrm{C})$ is the only exception. However, the domestic S\&C manufacturer in its applied research activities intensively develops new technologies. An optimized geometry, the application of the elastic elements in order to suppress the influence of variable vertical stiffness along the length of the switch and crossing, development of resistant materials for crossings, operational systems of the new generation, trough sleepers, monitoring systems for switches and movable frogs are the subject of research activities. A certain barrier to the development is the fact that high speed switches crossings is not easy tested by high-speed traffic in the Czech Republic.

Rail infrastructure administration faces with the development of rail defects which are usually connected to the increase of axle load and service speed. This can be remedied by increasing the rail steel quality, but in the surface layer resisting to fatigue loading. Thus new types of rail defects occur and appropriate preventive measures are looked for. The short pitch corrugation occurs in lower rail in tight curves which during wheelset passages besides vibrations causes also noise emission to the track vicinity. The only effective measure is the grinding or milling of the rails, which is very costly, requires service interruption and significantly reduces the life span of the rails. Effective measures may consist in an additional elasticity of the track or modification of the friction coefficient at the wheel-rail contact.

A significant part of the research work is aimed at the technology procedures and management of construction activities and maintenance work. Next subject of research works in this field is the development of advanced technological processes, rules of guarantee service and preventive maintenance strategy. Innovative and advanced technological procedures for

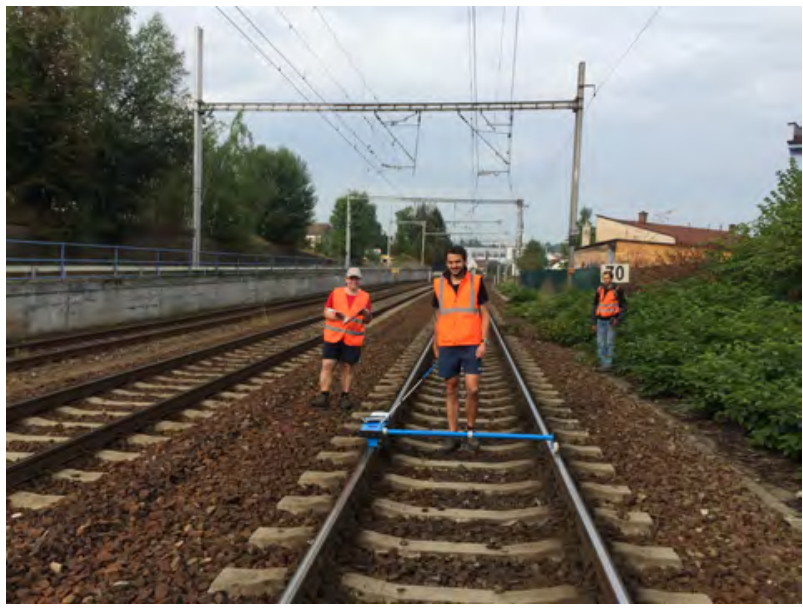

FIGURE 2. Investigation of rail corrugation by the Salamander device - Havlickuv Brod

maintenance and reconstruction of tracks, logistics issues, management and strategy of maintenance are developed.

\section{EnVironmental aspects of RAIL INFRASTRUCTURE}

Outcomes of research activities lead to the development of such infrastructure design, which also contributes to the reduction of negative impacts on the track vicinity, while solutions are being sought especially economically efficient from a lifecycle. cost perspective.

Railway tracks mainly in urban areas are perceived as a significant source of noise and vibrations. With a growing number of rail vehicles is adversely affected track vicinity. This negative influences were worse if any measures would not be used. The negative effects in some cases led to an idea to shift of tracks away of city centre, which, however, caused more difficult access of passengers to rail transport. This approach has been modified but this requires significant suppression of noise emission and vibrations propagation.

The noise caused by traffic not only adversely contributes to the emergence of various diseases. In principle, the noise generated by the rail transport can originate from wheel-rail contact, from vehicle engines and aggregates, which is significant for low service speed, and aerodynamic noise, which is significant for high service speed. The noise originated from wheelrail contact - rolling, squeezing, impact - is a common trouble for every rail vehicle which increases necessity to deal with this phenomenon. For this reason, not only an influence of parameters of rolling stock but also railway superstructure and substructure parameters and service conditions are investigated.

Vibrations that spread to building structures and building foundations are other loads. Vibrations are induced by dynamic effects from the train movement across the track irregularities. Characteristics of dynamic effects are related to number, weight and tech- 


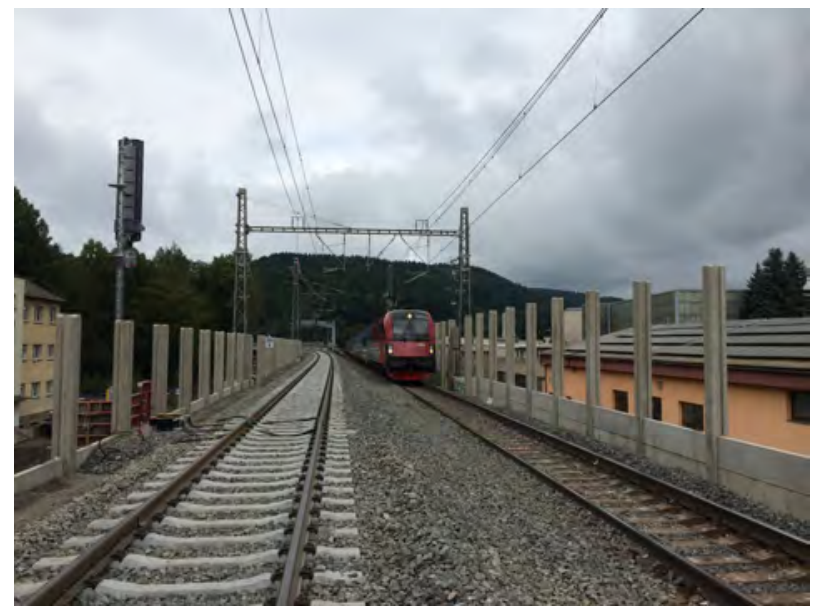

FIGURE 3. Installation of noise barriers in Usti nad Orlici

nical state of vehicles, service speed, running dynamic, track structure, track quality, track alignment, geological parameters, etc.

Preventive measures at the vibration source are most economical and most effective. If it is not possible to prevent structural vibrations at a source, then this source should be completely separated from adjacent structures. Special elastic rail fastening, under sleeper pads, under ballast mats, mass-spring systems or ballastless track are used as measures in track structure for vibration mitigation. Most types of these measures are available today as deliveries of recent applied research projects. Higher initial costs, which are usually outweighed by non-financial benefits, are currently an obstacle for immediate application of measures preventing vibration propagation.

\section{Conclusions}

Railway infrastructure administration put emphasize on research and development and implementation of research results into practice. It is considered as the fundamental way to increase the competitiveness of rail transport in the process of developing a sustainable and efficient transport.

Stakeholders (manufacturing companies, contractors, design companies, universities and research centres), which participate in the investment production and maintenance of the railway infrastructure in the Czech Republic, in the areas of infrastructure, energy, control, command and signalling, have jointed their efforts in The Czech Technology Platform. Its objective is the linkage of the scientific and technical potential of universities, research and project institutes together with the production potential of construction and manufacturing companies for the implementation of the following areas of activities [4]:

- Support of innovation and increase of competitiveness of the members of the Association

- Structuring and support of implementation of development, research and testing projects ensuring the existing production of the members of the Association with the requirements of the technical specifications of interoperability of the Trans-European railway system in the sub-systems of infrastructure, energy, control, command and signalling

- Acquiring of financial funds for implementation of these projects

- International (European) activities related to the creation of new regulations for construction, production and maintenance and related tests and evaluation of the railway industry

A variety of projects supported by national funding agencies, particularly the Technology Agency of the Czech Republic, Grant Agency of the Czech Republic or by the Ministry of Industry and Trade are solved nowadays. The project "Centre for efficient and sustainable transport infrastructure -CESTI" belongs among the most important projects, aimed in railway infrastructure. The Ministry of Education, Youth and Sports promotes the development of science and research projects through operational programs, an example is the project "Interoperability of Railway Infrastructure Competence Network (IRICoN)".

Projects can be also supported from different European funding programmes. The leading European companies in railway industry joined efforts in a joint undertaking Shift2Rail within the EU framework programme for research and innovation ? Horizon 2020. The Shift2Rail has just opened the first calls for project of applied research for its members and in the form of OpenCalls to non-members, too.

With respect to the current support of science and research projects at national and European level, with regard to available facilities of newly built research centres, excellent research teams, it can be assumed that research activities will achieve the objectives which were defined in the White Paper and further specified in the particular requirements of railway companies, contractors and manufacturers in the railway industry.

\section{ACKNOWLEDGEMENTS}

The article was processed under financial support of the project LO1408 "AdMaS UP - Advanced Building Materials, Structures and Technologies" supported by Ministry of Education, Youth and Sports within the "National Programme for Sustainability I".

\section{REFERENCES}

[1] European Commission. White Paper on Transport. Towards a competitive and resource efficient transport system., 2011. http://www.transforum-project.eu/ transforum/white-paper-on-transport.html.

[2] Union Internationale Chemins de fer (UIC). Rail and Sustainable Development, 2001. http://www.uic.org.

[3] Union Internationale Chemins de fer (UIC). High Speed Rail, Fast Track to Sustainable mobility, 2009. http://www.uic.org.

[4] www.sizi.cz, 2016. http://www.sizi.cz/introduction 